\title{
La intervención psicológica de la obesidad: desarrollo y perspectivas
}

\section{Psychological intervention of obesity: development and perspectives}

\author{
José I. Baile, María J. González-Calderón, Ruth Palomo y María F. Rabito-Alcón \\ Universidad a Distancia de Madrid, España
}

\begin{abstract}
Resumen: La obesidad es uno de los grandes problemas de salud en la actualidad, que afecta a millones de personas a lo largo de todo el mundo, lo que obliga a todos los profesionales de la salud a trabajar en su prevención y tratamiento. El papel de los psicólogos en la intervención de la obesidad ha sido tradicionalmente el tratamiento de las alteraciones psicológicas comórbidas, con las que suele cursar. Sin embargo, desde hace más de cuatro décadas, existen intervenciones psicológicas específicas para la obesidad, fundamentalmente desde una perspectiva cognitivo-conductual, las cuales no están aún muy extendidas. Este trabajo revisa la breve historia de la aproximación psicológica de la obesidad, analiza las evidencias empíricas que sustentan a los distintos programas de tratamiento, recopila cuáles son los componentes típicos de los enfoques cognitivo-conductuales, incluyendo varios protocolos de intervención, y pone en evidencia los retos que todavía le quedan a la psicología en la intervención de esta enfermedad.
\end{abstract}

Palabras clave: Obesidad, intervención psicológica, tratamiento cognitivo-conductual.

Abstract: Obesity is one of the greatest health problems today, affecting millions of people throughout the world, requiring all health professionals to work on its prevention and treatment. The role of psychologists in the intervention of obesity has traditionally been to treat the comorbid psychological disorders with which it is often associated. However, specific psychological interventions for obesity from a cognitive-behavioural perspective have been available for over four decades but are still not widespread. This paper reviews the brief history of psychological intervention of obesity, analyses the empirical evidence supporting these interventions, compiles the typical components of the cognitive-behavioural approach, including several intervention protocols, and highlights the challenges remaining for psychology in the intervention of this disease.

Keywords: Obesity, psychological intervention, cognitive-behavioural therapy.

Los autores de este artículo son todos ellos profesores de la Universidad a Distancia de Madrid.

La correspondencia sobre este artículo deberá ser enviada al primer autor al e-mail: joseignacio.baile@udima.es 
Según la Organización Mundial de la Salud (2018), la obesidad va a convertirse en la gran pandemia del siglo XXI. Según esta organización la obesidad se ha casi triplicado en todo el mundo desde 1975, ya en 2016, más de 1900 millones de adultos de 18 o más años tenían sobrepeso, de los cuales, más de 650 millones eran obesos; igualmente informa que la mayoría de la población mundial vive en países donde el sobrepeso y la obesidad se cobran más vidas de personas que la insuficiencia ponderal. A esto hay que sumarle el hecho de que la prevalencia de la obesidad infantil es actualmente muy elevada en todo el mundo (Alba-Martín, 2016; Baile, 2007; Karnik y Kanekar, 2012), lo que hace temer futuras generaciones de adultos obesos, dado que está constatado que presentar obesidad en la infancia incrementa el riesgo de padecerla en etapas evolutivas posteriores (Albañil et al., 2011).

La obesidad es una enfermedad asociada a una condición física, el exceso de grasa corporal, que conlleva importantes consecuencias no solo para la salud física (Moreno et al., 2000) sino también para la psíquica (Baile y González, 2011). En la sociedad actual, donde la delgadez constituye una cualidad personal positiva, los individuos con sobrepeso u obesidad sufren rechazo social, cuya interiorización incrementa la insatisfacción corporal y merma la autoestima (Fernández et al., 2019; Puhl y Heuer, 2010). Además del impacto en la calidad de vida de las personas que presentan sobrepeso u obesidad, últimamente se ha puesto de manifiesto el importante efecto que estas condiciones poseen en los costes sanitarios que deberán asumirse por los sistemas públicos y privados de salud para atender a millones de afectados, tanto a la hora de tratar la propia obesidad como las numerosas enfermedades asociadas (Olivia et al., 2008). Por ejemplo, en Estados Unidos se estimaba que el coste de la atención de los problemas sanitarios asociados a la obesidad constituía ya entonces el $1 \%$ de su producto interior bruto (Serrano, 2009).

Ante tamaño reto sanitario a nivel mundial, todos los profesionales de la salud están llamados a aportar su punto de vista relativo a los aspectos etiológicos, la propuesta de acciones preventivas, así como terapéuticas. Entre estos profesionales, los psicólogos han tenido, y deben seguir teniendo, un papel fundamental. El presente trabajo tiene como objetivos revisar el papel de la psicología en la intervención de la obesidad, realizando un breve recorrido histórico, analizando aquellas propuestas de intervención terapéutica con mayor aval empírico en la actualidad a partir de un análisis de las aportaciones de las investigaciones en la última década y describiendo las principales características de las mismas.

\section{Definición, evaluación y diagnóstico de la obesidad}

La obesidad se define como un exceso de grasa corporal en relación a ciertos límites, expresados en porcentaje de peso del tejido graso de un individuo, frente a su peso total. Tal y como recoge Moreno et al. (2000), un hombre presenta obesidad si su masa grasa supera el $25 \%$ de su peso corporal; en el caso de las mujeres, se considera obesidad si se supera el 32\% del mismo. Sin embargo, dada la dificultad para estimar con precisión la masa grasa de una persona, el indicador más utilizado para valorar el sobrepeso o la obesidad suele ser el índice de masa corporal (IMC), el cual se obtiene dividiendo el peso en kilogramos de un individuo entre su altura en metros al cuadrado. Si tenemos en cuenta dicho indicador, se ha llegado a un consenso en la comunidad científica (Rubio et al., 2007), en estimar que una persona presentará sobrepeso si su IMC se encuentra entre 24.9 y 29.9 , y obesidad, si este es igual o superior a 30.

La evaluación de la obesidad requiere la valoración de diferentes aspectos. Por una parte, los antropométricos, que proporcionarán una estimación de la situación corporal y la inclusión del individuo dentro de una u otra de las siguientes categorías: infrapeso, normopeso, sobrepeso u obesidad. Por otra parte, los hábitos alimentarios, de actividad física o sueño de quienes presentan esta enfermedad, entre otras áreas. Asimismo, en su valoración es imprescindible conocer las consecuencias psicosociales de la obesidad en el individuo, como el impato en sus relaciones sociales. Todos estos aspectos permitirán establecer un análisis funcional del origen y el mantenimiento de la misma. Igualmente, se considera necesaria la evaluación de los trastornos concomitantes, tanto biomédicos como psicológicos.

Respecto de su diagnóstico, es importante indicar que, si bien la obesidad es una enfermedad que puede ser diagnosticada como tal según la Clasificación Internacional de Enfermedades de la Organización Mundial de 
la Salud (10ª ed.; CIE-10; Organización Mundial de la Salud, 2010), la cual recoge cinco subtipos de obesidad, paradójicamente, no está considerada un trastorno psicológico o mental, ni tampoco un trastorno del comportamiento alimentario, según el Manual de Diagnóstico de Enfermedades Mentales de la Asociación Americana de Psiquiatría (5 ed.; DSM-5; American Psychiatric Association, 2013), a pesar del importante componente psicológico implicado en la etiología y mantenimiento de gran parte de los casos de obesidad.

\section{Origen y evolución de la aproximación psicológica a la obesidad}

No es hasta la segunda mitad del siglo XX cuando los profesionales de la salud comienzan a preocuparse por el tratamiento de la obesidad, condición física que en épocas pasadas no solo no era concebido como un problema de salud, sino que incluso se consideraba una manifestación de vitalidad, bienestar u opulencia. En concreto, los psicólogos han intervenido tradicionalmente tratando las comorbilidades de índole psicológica asociadas a la obesidad, trabajando la baja autoestima, la insatisfacción corporal o la ansiedad, entre otros aspectos. Sin embargo, ha sido menos patente su intervención sobre la obesidad en sí misma como objetivo prioritario de la terapia. El afianzamiento de las terapias conductuales, cognitivas y cognitivo-conductuales a lo largo de las décadas de los años 50 y 60 del siglo XX dio lugar a que un grupo de psicólogos plantearan el tratamiento de la obesidad mediante técnicas psicológicas exclusivamente, dando lugar al nacimiento de la terapia psicológica de la obesidad como rama específica de la psicoterapia. En este sentido, es necesario resaltar que cuando se sugiere a una persona llevar a cabo una dieta, lo que se le está proponiendo es realizar un cambio de hábitos o conductas, en este caso, en relación a su alimentación, lo cual podría decirse que es una prescripción psicológica, dado que la modificación de conducta es una de las áreas específicas dentro de la psicología. Esta matización es importante para reclamar, como psicológicas, muchas propuestas de intervención en casos de obesidad que históricamente se han venido realizando desde otras especialidades, incluso en aquellos casos en los que solo se incluía como plan de tratamiento seguir una determinada dieta.

En los años sesenta, Stuart (1967) fue uno de los primeros autores que publicaron un estudio referente a la intervención psicológica de enfoque conductual; en éste, ocho pacientes con obesidad consiguieron disminuir su peso tras un año de tratamiento con dicha terapia. El planteamiento básico del tratamiento conductual se fundamenta en que la obesidad es un desajuste de hábitos, los cuales son aprendidos por el individuo a lo largo de su historia vital y, son promovidos y mantenidos, tanto por sus circunstancias como por las propias consecuencias que conllevan, como sucede con cualquier otra conducta. Desde esta perspectiva, la terapia tiene como objetivo reeducar en dichos hábitos, trabajando con el sujeto y su contexto.

Pronto se detectó que el enfoque meramente conductual, dirigido fundamentalmente al control de la alimentación, era insuficiente, sobre todo a la hora de mantener los logros obtenidos o trabajar con ciertos individuos considerados "resistentes" a los tratamientos. Por este motivo, el modelo conductual fue enriqueciéndose con elementos procedentes del enfoque cognitivo y biológico, como por ejemplo, con la asunción de que existen tendencias genéticas que moldean ciertos fenotipos propensos a la obesidad, que hace que algunos sujetos sean especialmente resistentes a la reducción del peso mediante la mera modificación de hábitos. Todo ello ha dado lugar a un modelo cognitivo-conductual de tratamiento de la obesidad que entiende la necesidad de que otros profesionales también intervengan desde un planteamiento multidisciplinar. Foster, Markis y Bailer (2005) analizaron en detalle cómo se ha ido configurando históricamente dicho enfoque cognitivo-conductual. En los años 90 del siglo XX ya existían programas estandarizados de intervención desde este paradigma, por ejemplo el de Marcus (1993). En la actualidad, sabemos que el tratamiento de la obesidad desde este enfoque cognitivo-conductual es eficaz, como han recogido diferentes autores como Amigo (2013) o Wadden et al. (2005); estos últimos analizaron 42 trabajos de investigación publicados entre 1974 y 2002, ofreciendo un interesante resumen de las características de estas intervenciones psicológicas en cuanto a duración, pérdida de peso conseguida, grado de abandono, etc. Por ejemplo, observaron que la pérdida de peso promedio fue mejorando con el tiempo conforme los tratamientos se fueron enriqueciendo, de modo que en los últimos estudios dicha cifra se acercaba a 10,7 kg, frente a los $3,8 \mathrm{~kg}$ de $\operatorname{los}$ estudios llevados a cabo en 1974. Quizá ese aumento de pérdida de peso esté relacionado con la cada vez 
mayor duración de los programas de intervención cognitivo-conductual, que pasaron de un promedio de 8,4 semanas a 31,4 .

\section{¿Tratamiento psicológico, farmacológico o cirugía bariátrica?}

Dado que en la actualidad se dispone de diferentes modalidades de tratamiento eficaces para combatir la obesidad, la intervención psicológica, la farmacoterapia y la cirugía bariátrica, se plantea la cuestión de cuál debe ser el tratamiento de elección. Existe cierto consenso entre los especialistas, por un lado, en reservar las intervenciones farmacológicas y la cirugía para los casos más graves o resistentes; y por otro, en plantar la modificación de hábitos y del estilo de vida, que debería establecerse siempre desde un enfoque psicoterapéutico, como elemento común en todos los casos, y como tratamiento único en aquellos menos graves.

Mitchell y Cook (2005) recomiendan seguir las siguientes pautas a la hora de elegir el tratamiento más adecuado en un caso de sobrepeso u obesidad:

- Para casos de sobrepeso propone el tratamiento psicológico (modificación de hábitos, terapia conductual). Solo si un sujeto presenta sobrepeso de nivel II (IMC: 27-29.9) y trastornos biomédicos asociados, podría plantearse una aproximación farmacológica.

- Para casos de obesidad sugiere llevar a cabo un tratamiento psicológico y valorar el farmacológico en todos los casos. Asimismo, recomienda la cirugía bariátrica en casos de obesidad mórbida (IMC > 40) y obesidad tipo II (IMC: 35-39.9), si existen enfermedades comórbidas. De hecho, en la elección de la cirugía bariátrica suelen tenerse en cuenta, además del criterio de IMC, otros criterios biomédicos y psicológicos específicos (Rubio et al., 2004).

No obstante, todo planteamiento debe ajustarse siempre a la idiosincrasia del paciente, a sus singularidades. En función de las características del caso, pueden no seguirse estas recomendaciones a la hora de seleccionar la modalidad de tratamiento, e incluso puede emplearse una que suele reservarse para casos más graves, por ejemplo, cuando ha fracasado reiteradamente el tratamiento que le correspondía por sus características individuales. Esta sugerencia de flexibilidad en la elección del tratamiento es compartida por autores como Wadden y Osei (2002).

\section{Intervención psicológica de la obesidad en la actualidad.}

En los últimos años, se ha constatado que el enfoque cognitivo-conductual sigue siendo el tratamiento de referencia en las intervenciones psicológicas de la obesidad, aunque existen trabajos que ponen de manifiesto sus limitaciones, tal y como puede comprobarse en la tabla 1, que recoge los principales estudios procedentes de la revisión de investigaciones publicadas entre 2010-2019 que se ha llevado a cabo en este trabajo.

El tratamiento cognitivo-conductual empleado en todos los estudios analizados suele centrarse fundamentalmente en promover hábitos saludables de alimentación y actividad física. Los resultados indican que este tipo de intervención no solo reduce el peso u otras variables antropométricas, como el IMC, la masa grasa o la relación cadera-cintura (Miri et al., 2019), sino que también mejora aspectos de índole psicológica, como una mayor autoeficacia (Baños et al., 2019) o el bienestar psicológico (Roth et al., 2011), psicosociales, así como aquellos vinculados directamente con la salud, como la percepción de la calidad de vida (Weinreich et al., 2017). Algunos programas psicológicos no emplean la terapia cognitivo-conductual sino otro tipo de intervenciones, como la terapia de aceptación y compromiso, si bien suelen incluir técnicas de modificación de hábitos, propias de la intervención conductual, como las empleadas en el estudio de Tronieri et al. (2019), en el que se observaron mejorías en el peso de los pacientes pero también cambios a nivel cognitivo y psicosocial.

Respecto de la modalidad de tratamiento más eficaz, estudios recientes parecen constatar que la de tipo grupal no permite mantener los resultados en mayor medida que la terapia individual sola (Berk et al., 2018). Por otro lado, parece que el empleo de las nuevas tecnologías podría mejorar la eficacia de la terapia 
cognitivo-conductual (Baños et al., 2019); incluso el simple registro electrónico de la actividad física y la alimentación aumentan la eficacia de la intervención respecto de los tradicionales registros en papel (Oliver et al., 2013).

Varios estudios han analizado el papel de la intervención psicológica dentro de un tratamiento multidisciplinar para tratar la obesidad. Parece unánime el acuerdo respecto de los beneficios que conlleva dicha intervención en comparación con los tratamientos que no la incluyen, no solo en relación a la mayor pérdida de peso (McLean et al., 2016), sino a otros aspectos, por ejemplo, mejorando la calidad de vida de los pacientes (Freitas et al., 2007).

Respecto del mantenimiento de los resultados en relación a la pérdida de peso, parece que la intervención cognitivo-conductual es una terapia eficaz para mantener los logros durante al menos medio año (Madjd et al., 2019). No obstante, cuando se llevan a cabo seguimientos al cabo de varios años, el nivel de eficacia de los programas cognitivo-conductuales no parece ser muy elevado, ni superior a otras modalidades de intervención, como los programas de autoayuda (Cooper et al., 2010).

Tabla 1. Investigaciones sobre intervención psicológica de la obesidad realizados de 2010 a 2019

\begin{tabular}{|c|c|c|c|}
\hline Autores & $\begin{array}{l}\text { Población } \\
\text { objetivo }\end{array}$ & Tipo de terapia & Resultados principales \\
\hline Tronieri et al. (2019) & $\begin{array}{l}\text { Adolescentes con } \\
\text { obesidad }\end{array}$ & $\begin{array}{l}\text { Terapia de aceptación y com- } \\
\text { promiso (ACT) junto a la Mo- } \\
\text { dificación de Conducta (del } \\
\text { estilo de vida) }\end{array}$ & $\begin{array}{l}\text { Mejora del peso, el control cognitivo, } \\
\text { el hambre, la actividad física y varia- } \\
\text { bles psicosociales }\end{array}$ \\
\hline Baños et al. (2019) & $\begin{array}{l}\text { Niños con obesi- } \\
\text { dad }\end{array}$ & $\begin{array}{l}\text { Tratamiento cognitivo y con- } \\
\text { ductual (TCC) centrado en } \\
\text { promoción de alimentación } \\
\text { saludable y hábitos de activi- } \\
\text { dad física, respaldado por una } \\
\text { plataforma web }\end{array}$ & $\begin{array}{l}\text { Mayor autoeficacia, mejora del IMC } \\
\text { y el porcentaje de materia grasa. Las } \\
\text { tecnologías de la información y co- } \\
\text { municación mejoran la eficacia del } \\
\text { programa }\end{array}$ \\
\hline Madjd et al. (2019) & $\begin{array}{l}\text { Mujeres adultas } \\
\text { que habían perdi- } \\
\text { do al menos el } 10 \\
\% \text { de su peso }\end{array}$ & $\begin{array}{l}\text { Terapia cognitivo-conductual } \\
\text { (TCC) para el mantenimiento } \\
\text { de la reducción de peso }\end{array}$ & $\begin{array}{l}\text { La TCC es una herramienta eficaz } \\
\text { para el mantenimiento del peso du- } \\
\text { rante un período de } 24 \text { semanas en } \\
\text { personas que pierden peso con éxito }\end{array}$ \\
\hline Miri et al. (2019) & $\begin{array}{l}\text { Adolescentes con } \\
\text { sobrepeso }\end{array}$ & $\begin{array}{l}\text { Terapia cognitivo-conductual } \\
\text { para la reducción del peso }\end{array}$ & $\begin{array}{l}\text { Reducción significativa del IMC, la } \\
\text { relación cintura-cadera y la masa gra- } \\
\text { sa, así como una mejora de la salud } \\
\text { psicosocial, actividad física y calidad } \\
\text { de vida relacionada con la salud }\end{array}$ \\
\hline Berk et al. (2018) & $\begin{array}{l}\text { Pacientes con dia- } \\
\text { betes tipo II y obe- } \\
\text { sidad }\end{array}$ & $\begin{array}{l}\text { Tratamiento cognitivo-con- } \\
\text { ductual grupal para conseguir } \\
\text { mantener el peso a largo pla- } \\
\text { zo, tras una reducción inicial } \\
\text { por dieta }\end{array}$ & $\begin{array}{l}\text { El programa TCC grupal no fue supe- } \\
\text { rior a la atención habitual sola }\end{array}$ \\
\hline
\end{tabular}


Tabla 1 (Continuación). Investigaciones sobre intervención psicológica de la obesidad realizados de 2010 a 2019

\begin{tabular}{|c|c|c|c|}
\hline Autores & $\begin{array}{l}\text { Población } \\
\text { objetivo }\end{array}$ & Tipo de terapia & Resultados principales \\
\hline $\begin{array}{l}\text { Weinreich et al. } \\
\text { (2017) }\end{array}$ & $\begin{array}{l}\text { Adultos obesos } \\
\text { internados }\end{array}$ & $\begin{array}{l}\text { Tratamiento conductual, junto } \\
\text { a terapia nutricional y ejerci- } \\
\text { cio físico }\end{array}$ & $\begin{array}{l}\text { Reducción del IMC, mejora de los pa- } \\
\text { rámetros biomédicos y de la calidad } \\
\text { de vida percibida }\end{array}$ \\
\hline Freitas et al. (2017) & $\begin{array}{l}\text { Adolescentes con } \\
\text { obesidad }\end{array}$ & $\begin{array}{l}\text { Asesoramiento psicológico } \\
\text { dentro de un programa multi- } \\
\text { disciplinar }\end{array}$ & $\begin{array}{l}\text { La inclusión de un componente de } \\
\text { asesoramiento psicológico en el trata- } \\
\text { miento multidisciplinar proporciona } \\
\text { mayores beneficios (mejora la calidad } \\
\text { de vida) }\end{array}$ \\
\hline McLean et al. (2016) & $\begin{array}{l}\text { Adultos obesos } \\
\text { tratados en centro } \\
\text { especializado }\end{array}$ & $\begin{array}{l}\text { Intervención psicológica mul- } \\
\text { tidisciplinar }\end{array}$ & $\begin{array}{l}\text { La incorporación de una intervención } \\
\text { psicológica mantiene o mejora los } \\
\text { resultados de pérdida de peso en los } \\
\text { pacientes obesos }\end{array}$ \\
\hline Oliver et al. (2013) & $\begin{array}{l}\text { Adolescentes con } \\
\text { obesidad }\end{array}$ & $\begin{array}{l}\text { Intervención mediante regis- } \\
\text { tro de la actividad física y la } \\
\text { alimentación }\end{array}$ & $\begin{array}{l}\text { El registro es más eficaz si se emplea } \\
\text { un registro electrónico que si los da- } \\
\text { tos están en papel }\end{array}$ \\
\hline Lanza et al. (2012) & $\begin{array}{l}\text { Adultos obesos } \\
\text { sometidos a ciru- } \\
\text { gía bariátrica }\end{array}$ & $\begin{array}{l}\text { Tratamiento psicológico ge- } \\
\text { nérico de preparación para la } \\
\text { cirugía }\end{array}$ & $\begin{array}{l}\text { Mejora significativa tras la interven- } \\
\text { ción de: impulso por delgadez, buli- } \\
\text { mia, desconfianza interpersonal, asce- } \\
\text { tismo y conciencia interoceptiva }\end{array}$ \\
\hline Roth et al. (2011) & $\begin{array}{l}\text { Niños con obesi- } \\
\text { dad }\end{array}$ & $\begin{array}{l}\text { Tratamiento cognitivo-con- } \\
\text { ductual (TCC): nutrición, } \\
\text { actividad física, conducta ali- } \\
\text { mentaria y variables psicoso- } \\
\text { ciales }\end{array}$ & $\begin{array}{l}\text { Efectos moderados sobre el IMC y } \\
\text { efectos positivos en el bienestar psi- } \\
\text { cológico }\end{array}$ \\
\hline Cooper et al. (2010) & $\begin{array}{l}\text { Mujeres con obe- } \\
\text { sidad }\end{array}$ & $\begin{array}{l}\text { Tratamiento cognitivo-con- } \\
\text { ductual (TCC) para prevenir } \\
\text { las recaídas versus programa } \\
\text { mínimo de autoayuda }\end{array}$ & $\begin{array}{l}\text { En un seguimiento a tres años ambos } \\
\text { programas muestran poca eficacia en } \\
\text { el mantenimiento de los logros inicia- } \\
\text { les }\end{array}$ \\
\hline
\end{tabular}

\section{La intervención cognitivo-conductual de la obesidad}

A continuación, se resumen las características y modalidades de intervención cognitivo-conductual con mayor aval empírico en la actualidad a la hora de tratar casos de obesidad.

El tratamiento cognitivo-conductual de la obesidad no tiene un protocolo cerrado que deba aplicarse sistemáticamente a todos los pacientes, si algo caracteriza a este enfoque es la flexibilidad y adaptabilidad al caso concreto. No obstante, quienes lo emplean suelen seguir en el proceso terapéutico las siguientes etapas, 
las mismas que ante cualquier otro trastorno clínico o de la salud: evaluación/diagnóstico, análisis funcional, establecimiento de objetivos, aplicación de las técnicas de tratamiento, prevención de recaídas y seguimiento (Baile, 2011). Asimismo, la experiencia de varias décadas en el tratamiento de la obesidad ha permitido detectar ciertas áreas sobre las que intervenir, así como aquellas técnicas que resultan más eficaces y efectivas y, por ende, recomendables. En la tabla 2 se muestran los componentes específicos propuestos por diferentes autores para el tratamiento de la obesidad desde el enfoque cognitivo-conductual.

Tabla 2. Componentes de un programa de intervención cognitivo-conductual de la obesidad, según diversos autores

\section{Autores Componentes del programa cognitivo-conductual}

Baile y González (2013a) 1.- Promoción de la motivación para el cambio.

2.- Psico-educación sobre las causas generales de la obesidad y la posible etiología del caso concreto, sobre alimentación saludable (qué comer, cuándo y cómo) y sobre actividad física.

3.- Propuesta de régimen alimentario especial y un plan de actividad física.

4.-Intervención para incrementar el autocontrol.

5.- Intervención en las cadenas conductuales.

6.- Realización de autorregistros con intención terapéutica para modificar hábitos alimentarios y de actividad física.

7.- Empleo de técnicas generales de modificación de conducta para el incremento o reducción de conductas asociadas a los hábitos implicados.

8.- Modificación de estilos cognitivos asociados a los hábitos implicados.

9.- Promoción de las relaciones interpersonales: diseño de actividades gratificantes, entrenamiento en asertividad o habilidades sociales...

10.- Prevención de recaídas.

Foster et al. (2005)

1.- Promoción del autocontrol mediante autorregistros de hábitos de alimentación y de actividad física.

2.- Control de estímulos a través de la identificación de las señales asociadas a la ingesta.

3.- Educación sobre nutrición.

4.- Modificación de la conducta de comer (cómo, por ejemplo, comer lento).

5.- Realización de actividad física.

6.- Empleo de técnicas de restructuración cognitiva y de resolución de problemas.

Wadden, et al. (2005) 1.- Monitorización.

2.- Educación nutricional.

3.- Control de estímulos.

4.- Reducción de la ingesta.

5.- Promoción de la actividad física.

6.- Entrenamiento en la resolución de problemas.

7.- Prevención de recaídas.

Garaulety y Pérez de Here- 1 -- Control estimular.

dia (2010)

2.- Automonitorización.

3- Uso del refuerzo positivo.

4.- Restructuración cognitiva.

5.- Prevención de recaídas. 
Como puede deducirse de las propuestas incluidas en dicha tabla, las áreas habituales de tratamiento en obesidad se dirigen a los hábitos saludables (de alimentación y de actividad física) y a las variables cognitivas y sociales relacionadas con las conductas alteradas. En cuanto a las técnicas empleadas, estas son las habituales en este paradigma, destacando especialmente los autorregistros, las técnicas de control de contingencias, las técnicas de control estimular, el entrenamiento en resolución de problemas, la ruptura de cadenas conductuales y el entrenamiento en habilidades sociales, entre otras. La literatura científica actual nos proporciona descripciones detalladas que permiten aplicar con precisión dichas técnicas, como las de Gavino (2009) o Labrador (2009), en castellano.

Wadden y Osei (2002) recogieron las siguientes características de la terapia cognitivo-conductual que la literatura relaciona con una mayor eficacia en el objetivo de pérdida de peso:

- En cuanto a la frecuencia, el tratamiento es más eficaz si se realiza semanalmente.

- En cuanto a la duración, lo habitual es que dure entre 16 y 26 semanas, siendo muy recomendable que el periodo esté previamente definido.

- Es muy importante el planteamiento flexible del tratamiento, adaptando las técnicas al caso concreto.

- Es preferible un tratamiento grupal frente a uno individual. Asimismo, los grupos conviene que sean cerrados, sin incorporaciones de nuevos pacientes durante el curso del tratamiento.

\section{Protocolo grupal e individual de intervención psicológica de la obesidad}

A continuación, se exponen dos protocolos de intervención psicológica cognitivo-conductual de la obesidad, uno grupal y otro individual. Como ejemplo paradigmático de una intervención en formato grupal se recomienda la propuesta por Vera (1998), la cual se dirige a personas con sobrepeso u obesidad sin comorbilidades psicológicas importantes, a quienes se les haya recomendado una pérdida de peso menor de veinte kilos. El programa está previsto para grupos de no más de veinte sujetos y cuenta con la participación de dos terapeutas. Se estima que tiene una duración de doce sesiones. Los objetivos y estrategias que se van trabajando en cada sesión se exponen, muy sucintamente, a continuación:

- Sesión 1: Desarrollo del conocimiento entre los participantes, promoción de la motivación y psicoeducación sobre la obesidad. En esta sesión comienza el empleo de los autorregistros.

- Sesión 2: Evaluación de la motivación para perder peso. Se emplean autorregistros cognitivos y se valora la inclusión de un compañero.

- Sesión 3: Continuación del análisis de la motivación y la evaluación de aspectos cognitivos. Se elabora un análisis funcional y se plantea llevar a cabo un contrato conductual.

- Sesión 4: Promoción de la actividad física, trabajo cognitivo sobre pensamientos de autoimposición, control estimular y psicoeducación nutricional.

- Sesión 5: Continuación del control estimular y el trabajo cognitivo sobre las distorsiones cognitivas.

- Sesión 6: Modificación de hábitos de alimentación y de actividad física, así como trabajo cognitivo sobre las distorsiones.

- Sesión 7: Psicoeducación para modificar el estilo de alimentación y el tipo de vida sedentaria.

- Sesión 8: Promoción del cambio de la forma de comer, de rápido a lento, y continuación del trabajo cognitivo sobre las distorsiones.

- Sesión 9: Intervención sobre el estilo cognitivo caracterizado por el "tremendismo", aprendizaje de habilidades para comer fuera de casa y promoción del ejercicio físico.

- Sesión 10: Análisis y modificación de cadenas conductuales.

- Sesión 11: Prevención de recaídas, trabajando su afrontamiento.

- Sesión 12: Prevención de recaídas, trabajando su afrontamiento y el aprendizaje de estrategias de finalización de la terapia.

Para la exposición de un tratamiento individual recogemos la propuesta de Baile y González (2013b), que plantea sesiones clásicas individuales de terapia, las cuales se realizan de forma semanal al principio y más espaciadas en la parte final del tratamiento. Se proponen 20 sesiones a desarrollar a lo largo de unos 6-7 meses, 
si bien esta pauta debe interpretarse con mucha flexibilidad para adaptarse a la idiosincrasia del paciente. El enfoque es multidisciplinar, entendiendo que el psicólogo debe trabajar conjuntamente con, al menos, el médico general o especialista en endocrinología, así como con el nutricionista. La intervención se divide en las siguientes cuatro fases: 1) evaluación pre-tratamiento; 2) elaboración del análisis funcional y establecimiento de objetivos de terapia; 3) tratamiento; y 4) finalización y seguimiento. A modo de resumen, en la tabla 3 se indican los objetivos a trabajar en cada una de las sesiones.

Tabla 3. Objetivos del programa de intervención individual cognitivo-conductual para la obesidad propuesto por Baile y González (2013b)

\begin{tabular}{cl}
\hline Sesiones & Objetivos \\
\hline 1 & Concretar el motivo de consulta. \\
\hline 2 & Continuar la evaluación y descartar las alteraciones comórbidas. \\
\hline 3 & Explicar la primera propuesta de análisis funcional. \\
\hline 4 & Definir los objetivos, elegir las técnicas a emplear y promover la motivación para el cambio. \\
\hline 5 & Emplear psicoeducación y reestructuración cognitiva. \\
\hline $6-7$ & Continuar la reestructuración cognitiva. \\
\hline $8-11$ & Modificar hábitos alimentarios. \\
\hline $12-14$ & Modificar hábitos vinculados a la actividad física. \\
\hline 15 & Promover el autocontrol. \\
\hline 16 & Romper cadenas conductuales. \\
\hline $17-18$ & Promover habilidades sociales y actividades gratificantes. \\
\hline 19 & Educar en prevención de recaídas. \\
\hline
\end{tabular}

\section{Retos en la intervención psicológica en obesidad}

Son varios los retos a los que debe enfrentarse la intervención psicológica de la obesidad, algunos consustanciales a todas las aproximaciones de intervención en casos de obesidad, y otros específicos de la disciplina psicológica. Al igual que sucede en otros tratamientos de la obesidad, la terapia psicológica debe lograr cambios 
en el comportamiento del individuo luchando contra un contexto hostil y contradictorio, que promueve y facilita conductas obesogénicas; por tanto, la motivación para el cambio y las dificultades de intervención son mayores en comparación con otros problemas de salud. También comparte con otros tratamientos la dificultad de mantener los logros obtenidos; de hecho, un número elevado de pacientes que reciben terapia psicológica conductual o farmacológica, recuperan todo el peso perdido a los pocos años de finalizar el tratamiento, alcanzando cifras del 50\% según algunos autores (Devlin, Goldfein, Carino y Wolk, 2000; Kramer, Jeffery, Forster y Snell, 1989; Wadden, Sternberg, Letizia, Stunkard y Foster, 1989). Esto ha llevado a entender la obesidad como una enfermedad crónica, como podría ser la diabetes, que quizá requiera de una intervención de por vida. Dicha perspectiva debe ser integrada por la psicología de la salud para dar una respuesta terapéutica realista (Baile, 2019).

Entre los retos específicos de la intervención psicológica de la obesidad se encuentra, por una parte, terminar de perfilar los protocolos de intervención, depurando qué objetivos son cruciales en el tratamiento y qué técnicas son las que mayor evidencia o aval empírico presentan, al existir pocos trabajos de revisión y resultados contradictorios al respecto; por otra parte, es necesario dilucidar qué modalidad de intervención (solo psicológica, combinada con otros tratamientos o ninguna psicológica) resulta más adecuada a cada tipo de paciente en función de su edad, grado de sobrepeso u obesidad, así como otras características bio-psico-sociales; por último, otro contratiempo habitual es la presencia de comportamientos "compulsivos", pues muchos pacientes refieren episodios de atracones, en algunos casos, incluso cumplen todos los criterios para poder diagnosticar el trastorno por atracón (Baile, 2014; Yanovski, 2003). Este hecho convierte la intervención de la obesidad en una terapia de un trastorno del comportamiento alimentario, con las dificultades singulares que ello conlleva. De hecho, hasta la fecha no está claramente definido un protocolo de intervención en obesidad cuando existe un trastorno por atracón comórbido.

\section{Prevención de las recaídas y el aumento de peso}

El problema de las recaídas, y la consiguiente recuperación de peso, se sigue observando en alguna de las investigaciones revisadas, como el Cooper et al. (2010), lo cual hace cuestionar algunos de los planteamientos de las intervenciones psicológicas. Por una parte, esta la duda de si las estrategias de intervención están bien planteadas, dado que muchos trabajos se dirigen exclusivamente a proporcionar información sobre nutrición o actividad física, lo cual tiene un importante impacto inicial en el peso del paciente, pero no se dirigen a realizar modificaciones estables en los hábitos saludables de alimentación y ejercicio, que sí generarían no solo un impacto inicial, sino también cambios más permanentes en la reducción del peso. Por otra parte, existen intervenciones que tienen objetivos de reducción de peso excesivamente exigentes, asociados a alcanzar el peso considerado ideal por el paciente, o aquel sugerido por estándares estéticos, cuando reducciones no tan elevadas, del orden del 10\%, serían suficientes para mejorar los parámetros saludables y, respecto a lo que nos ocupa, constituiría un peso mucho más fácil de mantener en el tiempo.

Para mejorar el mantenimiento de los logros se han propuesto diversas estrategias; por ejemplo, Garaulet y Perez de Heredia (2010) apuntan las siguientes tras llevar a cabo una revisión de diferentes investigaciones: establecer sistemas de alimentación controlados varias veces a la semana, ser cautos en el establecimiento de los objetivos a alcanzar (proponiendo metas realistas), desarrollar programas de mantenimiento específicos (alargando la fase de seguimiento, haciendo uso de Internet, promoviendo mayor actividad física...) y aplicar nuevos enfoques procedentes de la terapia cognitiva de la obesidad, como los de Cooper y Fairburn (2001).

\section{Conclusiones}

La intervención de la obesidad es, y va a seguir siendo, uno de los grandes retos de los profesionales de la salud. La propagación de esta pandemia a nivel mundial es un hecho que ya preocupa a gobiernos e instituciones internacionales como la OMS. Por ello, es crucial que la Psicología como disciplina reclame su papel en el afrontamiento y solución de este problema. 
Desde hace unas décadas, los psicólogos ya no se dedican exclusivamente a tratar los trastornos psicológicos comórbidos que acompañan a los pacientes con sobrepeso u obesidad, sino que han propuesto estrategias de intervención para el tratamiento específico de ambos. La justificación de este avance es clara, pues la obesidad en prácticamente la mayoría de los casos está producida y mantenida por una descompensación de hábitos y, por tanto, reconducir dicha situación pasa por modificar estos. La disciplina que tiene entre sus objetivos analizar y modificar conductas es la psicología, la cual dispone de herramientas claramente validadas para conseguir que un paciente inicie conductas nuevas, modifique la intensidad o frecuencia de otras, o elimine aquellas que se consideran inadecuadas o nocivas para un individuo. Por ello, el psicólogo es un elemento clave en el tratamiento de la obesidad, y en muchos casos, debería incluso ser el central. Que los psicólogos sean profesionales de referencia en el tratamiento de un trastorno asociado al comportamiento alimentario, y que otros profesionales realicen tareas complementarias, es algo que diversos investigadores en el campo de los trastornos del comportamiento alimentario ya han venido reclamando, como ha quedado patente en las palabras del experto en la materia F. G. Fairburn en relación a la bulimia nerviosa, tal y como recoge Raich (2011). Asimismo, en el campo de la prevención, la psicología también tiene larga experiencia en el desarrollo de programas de promoción de la salud, por lo que debería ser tenida en cuenta en la elaboración de estrategias preventivas de la obesidad, campo en el que ya se han realizado propuestas interesantes al respecto, por ejemplo, en el ámbito de la obesidad infantil (Aguilar et al., 2011; Vela et al., 2009).

Con objeto de analizar el papel de la psicología en la intervención de la obesidad, en este trabajo se ha realizado una revisión de las investigaciones al respecto llevadas a cabo en la última década. De la misma se puede concluir que el enfoque más utilizado en las intervenciones psicológicas en obesidad es el enfoque cognitivo-conductual, si bien autores como Volery, Bonnemain, Latino, Ourrad y Perroud (2015) han comprobado la utilidad de otros enfoques, como la hipnosis y la atención plena, que se proponen para el manejo de las emociones y el estrés relacionadas con la obesidad; la terapia EMDR (desensibilización y reprocesamiento del movimiento ocular) la cual parece mejor que otros tipos de terapias si está involucrado el síndrome de estrés postraumático; y la terapia familiar, que puede estar indicada cuando el entorno sociofamiliar se ve afectado.

Los estudios son, en general, coincidentes en hallar eficacia de las intervenciones psicológicas, tanto para reducir el peso como para conseguir mantener los resultados, aunque es en este último aspecto donde los resultados son menos unánimes y se encuentran discrepancias entre los trabajos, como reflejan los estudios de Berk et al. (2018) y Cooper et al. (2010). Tal y como se ha apuntado, la falta de inclusión de estrategias específicas para la prevención de recaídas en los programas de tratamiento o perseguir un objetivo de peso excesivamente exigente, podrían subyacer a la recuperación del peso o la alta tasas de recaídas.

No obstante, resulta difícil realizar comparaciones entre las distintas investigaciones analizadas que permitan obtener conclusiones comunes, dado que no existe una homogeneidad en su metodología ni en la forma de comunicar los resultados. Así, las principales limitaciones encontradas a la hora de analizar los estudios revisados fueron las siguientes: no siempre se define claramente qué tipo de intervención psicológica se ha realizado con los pacientes, ni cuáles de las múltiples técnicas que incluye la terapia cognitivo-conductual se han utilizado exactamente en cada estudio. Por otro lado, los objetivos de tratamiento no son siempre los mismos; en unos casos se busca la modificación de hábitos, en otros la psicoeducación, en otros cambios en el peso o IMC, etc. Por último, señalar que las muestras varían también de manera significativa de unos estudios a otros, tanto en edad (de muestra infantil a adulta) como en tipología, pues el nivel de severidad de la obesidad es diferente, empleándose incluso muestras de centros de internamiento. Todo ello complica de manera significativa la generalización de los resultados.

En este artículo se ha puesto de manifiesto que la terapia psicológica de la obesidad tiene ya cierta trayectoria histórica, dispone de estudios sobre su eficacia y ha sido capaz de elaborar programas de intervención, más o menos sistemáticos, tanto en formato grupal como individual. Ello debe sustentar la exigencia reclamada por los psicólogos previamente apuntada acerca de que la psicología debe ser tenida en cuenta en el tratamiento de la obesidad. No obstante, también se han destacado los retos que le quedan a esta disciplina por afrontar en la consecución de este objetivo, al igual que les sucede a otras disciplinas del ámbito de la salud, algunos metodológicos, como puede ser la depuración y concreción de los protocolos de intervención, y otros teóricos, como puede ser la progresiva asunción de la obesidad como una enfermedad crónica y lo que la misma conlleva. 
Con objeto de obtener datos más contundentes, y con utilidad práctica, de las investigaciones sobre intervenciones psicológicas en obesidad, sería recomendable un consenso entre la comunidad científica sobre cuáles son los objetivos específicos que deben trabajarse (si una reducción de peso o una mejora de hábitos, por ejemplo) o el protocolo base tanto para la fase de intervención como la de seguimiento y prevención de caídas .

\section{Referencias}

Aguilar, E., González, C., García, P. A., García, J., Álvarez, C. A., Padilla, J. L., González-Mendoza, J. L. y Ocete, E. (2011). Obesidad de una población de escolares de Granada: evaluación de la eficacia de una intervención educativa. Nutrición Hospitalaria, 26(3), 636-641. https://doi.org/10.3305/nh .2011.26.3.5195

Alba-Martín, R. (2016). Prevalencia de obesidad infantil y hábitos alimentarios en educación primaria. Enfermería Global, 15(2), 40-62. https://doi.org/10.6018/eglobal.15.2.212531

Albañil, M. R., Rogero, M. E., Sánchez, M., Olivas, A., Rabanal, A. y Sanz, M. T. (2011). Riesgo de mantener obesidad desde la infancia hasta el final de la adolescencia. Pediatría Atención Primaria, 13(50). https://doi. org/10.4321/S1139-76322011000200003

American Psychiatric Association. (2013). Diagnostic and statistical manual of mental disorders (5 ${ }^{\mathrm{a}}$ Ed.). https://doi.org/10.1176/appi.books.9780890425596

Amigo, I. (2013). Intervención psicológica en obesidad. [Recensión]. Psicothema, 25, 415.

Baile, J. I. (2007). Obesidad infantil, ¿qué hacer desde la familia? Editorial Síntesis.

Baile, J. I. (2011). Intervención y tratamiento psicológico. Editorial UDIMA.

Baile, J. I. (2014). Trastorno por atracón: reconocido oficialmente como el nuevo trastorno del comportamiento alimentario. Revista Médica de Chile, 142(1), 128-129. https://doi.org/10.4067/S0034-98872014000100022

Baile, J. I. (2019). ¿Por qué no somos eficaces en el tratamiento de la obesidad? La necesaria aportación de la psicología. Medicina Clínica 152(2), 72-74. https://doi.org/10.1016/j.medcli.2018.06.003

Baile, J. I. y González, M. J. (2011). Comorbilidad psicopatológica en obesidad. Anales del Sistema Sanitario Navarro, 34(2), 253-261. https://doi.org/10.4321/S1137-66272011000200011

Baile, J. I. y González, M. J. (2013a). Intervención psicológica en obesidad. Editorial Pirámide.

Baile, J. I. y González, M. J. (2013b). Tratando la obesidad, técnicas y estrategias terapéuticas. Editorial Pirámide.

Baños, R. M., Oliver, E., Navarro, J., Vara, M. D., Cebolla, A., Lurbe, E., Álvarez, J., Torró, M.I. y Botella, C. (2019). Efficacy of a cognitive and behavioral treatment for childhood obesity supported by the ETIOBE web platform. Psychology, Health \& Medicine, 24(6), 703-713. https://doi.org/10.1080/13548506.2019.15 66622

Berk, K. A., Buijks, H. I., Verhoeven, A. J., Mulder, M. T., Özcan, B., van’t Spijker, A., Timman, R., Busschbach, J. J. y Sijbrands, E. J. (2018). Group cognitive behavioural therapy and weight regain after diet in type 2 diabetes: results from the randomised controlled POWER trial. Diabetologia, 61(4), 790-799. https://doi. org/10.1007/s00125-017-4531-9

Cooper, Z., Doll, H. A., Hawker, D. M., Byrne, S., Bonner, G., Eeley, E., O’Connor, M. E. y Fairburn, C. G. (2010). Testing a new cognitive behavioural treatment for obesity: A randomized controlled trial with threeyear follow-up. Behaviour Research and Therapy, 48(8), 706-713. https://doi.org/10.1016/j.brat.2010.03.008

Cooper, Z. y Fairburn, C.G. (2001) A new cognitive behavioral approach to the treatment of obesity. Behaviour Research and Therapy, 39, 499-511. https://doi.org/10.1016/S0005-7967(00)00065-6

Devlin, M. J., Goldfein, J. A., Carino, J. S. y Wolk, S. L. (2000). Open treatment of overweight binge eaters with phentermine and fluoxetine as an adjunct to cognitive-behavioral therapy. International Journal of Eating Disorders, 28, 325-332. https://doi.org/10.1002/1098-108X(200011)28:3<325::AID-EAT10>3.0.CO;2-3

Fernández, M. G., Feu, S. M., Suárez, M. R. y Suárez, Á. M. (2019). Satisfaction, index of body mass and its influence on physical self-concept. Revista Española de Salud Pública, 93.

Foster, G. D., Makris, A. P. y Bailer, B. A. (2005). Behavioral treatment of obesity. American Journal of Clinical Nutrition, 82, 230-235. https://doi.org/10.1093/ajen/82.1.230S 
Freitas, C. R., Gunnarsdottir, T., Fidelix, Y. L., Tenório, T. R., Lofrano-Prado, M. C., Hill, J. O. y Prado, W. L. (2017). Effects of a psychological intervention on the quality of life of obese adolescents under a multidisciplinary treatment. Jornal de Pediatria, 93(2), 185-191. https://doi.org/10.1016/j.jped.2016.05.009

Garaulet, M. y Pérez de Heredia, F. (2010). Behavioural therapy in the treatment of obesity (II): role of the Mediterranean diet. Nutrición Hospitalaria, 25, 9-17.

Gavino, A. (2009). Guía de técnicas de terapia de conducta. Editorial Pirámide.

Karnik, S. y Kanekar, A. (2012). Childhood obesity: a global public health crisis. International Journal of Preventive Medicine, 3, 1-7.

Kramer, F. M., Jeffery, R. W., Forster, J. L. y Snell, M. K. (1989). Long-term follow-up behavioral treatment for obesity: patterns of weight regain among men and women. International Journal of Obesity and Related Metabolic Disorders, 13, 123-136.

Labrador, F. J. (2009). Técnicas de modificación de conducta. Editorial Pirámide.

Lanza, L., Linda, M., Carrard, I., Reiner, M. y Golay, A. (2012). Psychological preparation for gastric bypass surgery. Revue Medicale Suisse, 8(334), 692-695.

Madjd, A., Taylor, M. A., Delavari, A., Malekzadeh, R., Macdonald, I. A. y Farshchi, H. R. (2019). Effects of cognitive behavioral therapy on weight maintenance after successful weight loss in women; a randomized clinical trial. European Journal of Clinical Nutrition. https://doi.org/10.1038/s41430-019-0495-9

Marcus, M. D. (1993). Obesidad. En M. Hersen y C.G. Last (Ed.), Manual de casos de terapia de conducta (pp. 149-161). Desclée de Brouwer.

McLean, R. C., Morrison, D. S., Shearer, R., Boyle, S. y Logue, J. (2016). Attrition and weight loss outcomes for patients with complex obesity, anxiety and depression attending a weight management programme with targeted psychological treatment. Clinical Obesity, 6(2), 133-142. https://doi.org/10.1111/cob.12136

Miri, S. F., Javadi, M., Lin, C. Y., Griffiths, M. D., Björk, M. y Pakpour, A. H. (2019). Effectiveness of cognitive-behavioral therapy on nutrition improvement and weight of overweight and obese adolescents: A randomized controlled trial. Diabetes \& Metabolic Syndrome: Clinical Research \& Reviews, 13(3), 2190-2197. https://doi.org/10.1016/j.dsx.2019.05.010

Mitchell, J. E. y Cook, T. (2005). Evaluación de la conducta del paciente y perspectiva de la intervención. Clínicas Psiquiátricas de Norteamérica, 28, 105-16.

Moreno, B., Monereo, S. y Álvarez, J. (2000). Obesidad, la epidemia del siglo XXI. Días de Santos.

Oliva, J., González, L., Labeaga, J. M. y Álvarez, C. (2008). Salud pública, economía y obesidad: el bueno, el feo y el malo. Gaceta Sanitaria, 22, 507-510. https://doi.org/10.1016/S0213-9111(08)75346-9

Oliver, E., Baños, R. M., Cebolla, A., Lurbe, E., Alvarez-Pitti, J. y Botella, C. (2013). An electronic system (PDA) to record dietary and physical activity in obese adolescents; data about efficiency and feasibility. $\mathrm{Nu}$ trición Hospitalaria, 28(6), 1860-1866. https://doi.org/10.3305/nutrhosp.v28in06.6784

Organización Mundial de la Salud (2010). International statistical classification of diseases and related health problems (10a ed.). https://icd.who.int/browse10/2019/en

Organización Mundial de la Salud - OMS (2018). Obesidad y sobrepeso. [Nota descriptiva]. https://www.who. int/es/news-room/fact-sheets/detail/obesity-and-overweight

Puhl, R. M. y Heuer, C. A. (2010). Obesity stigma: important considerations for public health. American Journal of Public Health, 100(6), 1019-1028. https://doi.org/10.2105/AJPH.2009.159491

Raich, R. M. (2011). Anorexia, bulimia y otros trastornos alimentarios. Editorial Pirámide.

Roth, B., Munsch, S. y Meyer, A. H. (2011). Long-term evaluation of a psychological training for obese children and their parents (TAKE). Praxis der Kinderpsychologie und Kinderpsychiatrie, 60(4), 304-321. https://doi.org/10.13109/prkk.2011.60.4.304

Rubio, M. A., Martínez, C., Vidal, O., Larrad, A., Salas-Salvadó, J., Pujol, J., ... Moreno, B. (2004). Documento de consenso sobre cirugía bariátrica. Revista Española de Obesidad, 4, 223-249.

Rubio, M., Salas-Salvadó, J., Barbany, M., Moreno, B., Aranceta, J., Bellido, D., ... Vidal, J. (2007). Consenso SEEDO 2007 para la evaluación del sobrepeso y la obesidad y el establecimiento de criterios de intervención terapéutica. Revista Española de Obesidad, 3, 7-48.

Serrano, M. (2009). La pandemia de obesidad y sus consecuencias metabólicas. Real Academia Nacional de Medicina. 
Stuart, R. B. (1967). Behavioral control of overeating. Behavior Research and Therapy, 5, 357-365. https://doi. org/10.1016/0005-7967(67)90027-7

Tronieri, J. S., Wadden, T. A., Leonard, S. M. y Berkowitz, R. I. (2019). A pilot study of acceptance-based behavioural weight loss for adolescents with obesity. Behavioural and Cognitive Psychotherapy, 1-11. https:// doi.org/10.1017/S1352465819000262

Vela, A, García., Z, Goñi. A., Suinaga., I., Aguayo, A., Rica, I. y Martul, P. (2009). Plan de intervención en la prevención de obesidad infantil "Erosotasuna Zainduz”. Revista Española de Obesidad, 7(2), 91-96.

Vera, M. N. (1998). Evaluación y tratamiento de la obesidad. En V. Caballo (Dir.). Manual para la intervención cognitivo-conductual de los trastornos psicológicos (Vol. 2, pp. 217-248). Siglo XXI.

Volery, M., Bonnemain, A., Latino, A., Ourrad, N. y Perroud, A. (2015). Obesity psychological treatment: beyond cognitive and behavioral therapy. Revue Medicale Suisse, 11(467), 704-708.

Wadden, T. A., Crerand, C. E. y Brock, J. (2005). Tratamiento conductual en la obesidad. Clínicas Psiquiátricas de Norteamerica, 28, 151-170. https://doi.org/10.1016/j.psc.2004.09.008

Wadden, T. A. y Osei, S. (2002). The treatment of obesity: an overview. En T. A. Wadden y A. J. Stunkard, (Eds.), Handbook of Obesity Treatment (pp. 229-248). Guilford Press.

Wadden, T.A., Sternberg, J.A., Letizia, K.A., Stunkard, A.J. y Foster, G.D. (1989). Treatment of obesity by very calorie diet, behaviour therapy, and their combination: a five-year perspective. International Journal of Obesity, 13, 2, 39-46.

Weinreich, T., Filz, H. P., Gresser, U. y Richartz, B. M. (2017). Effectiveness of a four-week diet regimen, exercise and psychological intervention for weight loss. Journal of Clinical and Diagnostic Research, 11(3), LC20-LC24. https://doi.org/10.7860/JCDR/2017/24112.9553

Yanovski, S. Z. (2003). Binge eating disorder and obesity in 2003: could treating an eating disorder have a positive effect on the obesity epidemic? International Journal of Eating Disorders, 34, 117-120. https://doi. org/10.1002/eat.10211

Artículo recibido: 10/04/2019

Artículo aceptado: 30/01/2020 